\title{
Bipartite entanglement, spherical actions and geometry of local unitary orbits
}

\author{
Alan Huckleberry ${ }^{1}$, Marek Kuśs ${ }^{2}$, Adam Sawicki ${ }^{2,3}$ \\ 1 Fakultät für Mathematik, Ruhr-Universität Bochum, D-44780 Bochum, Germany \\ 2 Center for Theoretical Physics, Polish Academy of Sciences, Al. Lotników 32/46, 02-668 Warszawa, \\ Poland \\ 3 School of Mathematics, University of Bristol, University Walk, Bristol BS8 1TW, UK
}

Received: date / Accepted: date

\begin{abstract}
We use the geometry of the moment map to investigate properties of pure entangled states of composite quantum systems. The orbits of equally entangled states are mapped by the moment map onto coadjoint orbits of local transformations (unitary transformations which do not change entanglement). Thus the geometry of coadjoint orbits provides a partial classification of different entanglement classes. To achieve the full classification a further study of fibers of the moment map is needed. We show how this can be done effectively in the case of the bipartite entanglement by employing Brion's theorem. In particular, we presented the exact description of the partial symplectic structure of all local orbits for two bosons, fermions and distinguishable particles.
\end{abstract}

\section{Introduction. Statement of results}

Quantum correlations among parts of a multicomponent (multipartite) composite quantum systems are usually described and investigated employing tools of multilinear algebra. This seems to be a natural method since at the most elementary level pure nonentangled states are simple tensors in the tensor product of Hilbert spaces of subsystems of an $L$-partite system,

$$
\mathcal{H}=\mathcal{H}_{1} \otimes \cdots \mathcal{H}_{L},
$$

whereas various degrees of entanglement can be characterized by quantifying (in some precisely defined sense) the "departure" of a state from the simple tensor structure.

Behind these algebraic definitions one finds interesting geometry. In [1] and [2] we showed how some methods of symplectic geometry can be used to describe and quantify entanglement. Two basic observations are crucial. From a physical point of view entanglement properties remain unchanged under the action of "local" unitary group $K=U\left(\mathcal{H}_{1}\right) \times \ldots \times U\left(\mathcal{H}_{L}\right)$ which reflects the physically obvious statement that entanglement can not be changed by non-dissipative quantum operations confined to 
subsystems. It follows thus that a classification and description of orbits of $K$ in the space of states (which, as quantum mechanics demands, is not $\mathcal{H}$ itself, but rather its projectivization $\mathbb{P}(\mathcal{H})$ ) leads to a classification of various degrees of entanglement. The second ingredient of this approach is an observation that both the space of states $\mathbb{P}(\mathcal{H})$ and the dual of the Lie algebra $\mathfrak{k}^{*}$ of $K$ possesses natural Poisson structures intertwined by the $K$-equivariant moment map $\mu$ which sends $K$-orbits in $\mathbb{P}(\mathcal{H})$ onto coadjoint orbits. Coadjoint orbits are in fact symplectic manifolds but since the moment map is not diffeomorphic the symplectic form on $\mathbb{P}(\mathcal{H})$ is, in general, degenerate on $K$-orbits. It is not the case only for the unique orbit of non-entangled states and the degree of degeneracy of the symplectic form on an orbit can be used as a measure of entanglement [1]. The moment map can be used as a tool for a partial classification of $K$-orbits in $\mathbb{P}(\mathcal{H})$ since it maps them onto coadjoint orbits which can be easily classified by their intersections with the dual to the Cartan subalgebra of the Lie algebra $\mathfrak{k}$ of $K$. Such a classification would give an effective answer to the problem of the "local unitary equivalence of states" (see [3]), i.e. whether two states can be mutually connected by non-dissipative quantum operations restricted to subsystems. The problem, important from the experimental point of view when the possibility of obtaining requested states from those which are available, translates thus into deciding if two states belong to the same $K$-orbit. Obviously, since the moment map is not a diffeomorphism one does not obtain a satisfactory classification of $K$-orbits in $\mathbb{P}(\mathcal{H})$ by mapping them into coadjoint ones. It may happen that many (in fact a continuous family of) orbits are mapped onto a single coadjoint orbit. Hence, for a full characterization a deeper insight into the structure of fibers of the moment map is needed. We will show how to do it for $L=2$. We will also show on examples how the structure of fibers and, consequently, classification of orbits become more complicated for systems with more parts.

The above outlined formulation of the problem allows for generalizations to cases when the underlying Hilbert space $\mathcal{H}$ does not have a structure of the full tensor product, but eg., is its symmetric, $S^{2} \mathcal{H}_{1}$, or antisymmetric, $\bigwedge^{2} \mathcal{H}_{1}$, part as in the cases of two indistinguishable particles (bosons and fermions). Since all three cases bear significant similarities we will consider them in parallel whenever possible.

Our results are stated precisely in the last section of the paper. For those readers most interested in the qualitative aspects of these results we state them here without technical details of proofs.

The goal of our work is to give an exact description of the partial symplectic structure of all $K$-orbits in $M=\mathbb{P}(\mathcal{H})$ and $L=2$. To do this we first observe that it follows from Brion's Theorem [5] that the moment map $\mu: M \rightarrow \mathfrak{k}^{*}$ parameterizes the $K$-orbits in $M$ in the sense that it maps the set of $K$-orbits in $M$ bijectively onto the set of $K$-orbits in its image. It is well-known that, modulo the action of the Weyl-group, this image is $K . P$ where $P$ a convex region in $\mathfrak{t}^{*}$. Here $\mathfrak{t}$ is the Lie algebra of diagonal matrices in $\mathfrak{k}=\mathfrak{s u}_{N}$ and $\mathfrak{t}^{*}$ is embedded in $\mathfrak{k}$ via the invariant form $\langle A, B\rangle=\operatorname{Tr}(A B)$. In fact $P$ intersects each orbit of $K$ in an orbit of the Weyl group so that, modulo the Weyl group, $P$ parameterizes the orbits. We give an exact description of $P$ as a certain probability polyhedron.

We also determine a real algebraic set $\Sigma_{\mathbb{R}}^{+}$in $M$, defined by linear algebraic equations and inequalities, which parameterizes the $K$-orbits in $M$ and which is mapped onto a fundamental region of the Weyl-group in $P$ by the moment map. Every element $x$ of $\Sigma_{\mathbb{R}}^{+}$determines in a simple way a vector $d=\left(d_{1}, \ldots, d_{k}\right)$ of positive integers which completely determines the moment map image $\mu(x)$ as a flag manifold $F\left(d_{1}, \ldots, d_{k}\right)$. We also exactly describe the fiber $\mathcal{F}_{x}=\mu^{-1}(\mu(x))$ of the moment map. It is the fiber 
of the homogeneous fibration $K / K_{x} \rightarrow K / K_{\mu(x)}$ which in fact (up to very simple finite-coverings) is a product of certain symmetric spaces. In the case of bosons it is the product of a torus and a number (depending on $d$ and the degeneracy) of symmetric spaces of the form $\mathrm{SU}_{m} / \mathrm{SO}_{m}$. The case of fermions is analogous, except that the symmetric spaces are of the form $\mathrm{SU}_{m} / \mathrm{USp}_{m}$.

To make the paper reasonably concise and, simultaneously, accessible to readers not familiar with the whole needed background material we provide in Appendix comprehensive outline of the most important results concerning spherical varieties. On the other hand we decided that the main text of the paper should remain self-contained hence the needed definitions and statements, even if they concern the background material, are often accompanied by shortened explanations and arguments fuller versions of which are given in the Appendix.

\section{Local unitary actions in spaces of states}

Let $\mathcal{H}_{1} \cong \mathbb{C}^{N}$, the $N$-dimensional complex vector space equipped with a scalar product $\langle\cdot \mid \cdot\rangle$ be the one particle Hilbert space. Let $G=\mathrm{SL}_{\mathbb{C}}\left(\mathcal{H}_{1}\right)$ be the special linear group and and $K=S U\left(\mathcal{H}_{1}\right)$ be the subgroup of unitary transformations in it. The two particle Hilbert spaces for bosons, fermions and distinguishable particles are, respectively, the symmetric and antisymmetric part of the tensor product of two copies of $\mathcal{H}_{1}$ and the full tensor product itself,

$$
\begin{aligned}
\mathcal{H}_{B} & =S^{2} \mathcal{H}_{1}, \text { bosons } \\
\mathcal{H}_{F} & =\bigwedge^{2} \mathcal{H}_{1}, \text { fermions } \\
\mathcal{H}_{D} & =\bigotimes^{2} \mathcal{H}_{1}=\mathcal{H}_{B} \oplus \mathcal{H}_{F}, \text { distinguishable particles }
\end{aligned}
$$

We have the natural action of $G_{D}=G \times G$ and $K_{D}=K \times K$ on $\mathcal{H}_{D}$ given by

$$
\left(U_{1}, U_{2}\right) \cdot(v \otimes w)=U_{1} \otimes U_{2}(v \otimes w)=\left(U_{1} v \otimes U_{2} w\right) .
$$

The diagonal action of $G$ and $K$ on $\mathcal{H}_{D}$, i.e.

$$
U \cdot(v \otimes w)=U \otimes U(v \otimes w)=U v \otimes U w,
$$

induces the action of $G, K$ on $\mathcal{H}_{B}$ and $\mathcal{H}_{F}$. In the following we will denote by $v \vee w:=$ $v \otimes w+w \otimes v$ and $v \wedge w:=v \otimes w-w \otimes v$ the symmetric and antisymmetric tensor products. It will be convenient to regard the tensors at hand as matrices. Therefore we let $\left\{e_{1}, \ldots, e_{N}\right\}$ be an orthonormal basis of $\mathcal{H}_{1}$, define $H$ (the complex torus) to be the subgroup of $G$ of diagonal matrices and let $T:=H \cap K$ be the corresponding real torus consisting of unitary diagonal matrices with determinant equal to one. Observe that $\left\{e_{i j}^{s}:=e_{i} \vee e_{j} ; 1 \leq i, j \leq N\right\}$ and $\left\{e_{i j}^{a}:=e_{i} \wedge e_{j} ; i<j\right\}$ are orthogonal bases of $\mathcal{H}_{B}$ and $\mathcal{H}_{F}$ respectively. We can regard any tensor $v$ in $\mathcal{H}_{D}$ as $N \times N$-matrix $C_{v}$.

$$
v=\sum_{i, j}\left(C_{v}\right)_{i j} e_{i} \otimes e_{j} .
$$

The matrix $C_{v}$ is symmetric for symmetric tensors and antisymmetric for antisymmetric ones hence we can write analogous formulae for $v \in \mathcal{H}_{B}$ and $v \in \mathcal{H}_{B}$ substituting $e_{i j}^{s}$ 
and $e_{i j}^{a}$ in place of $e_{i} \otimes e_{j}$ completing thus the identification of tensors with matrices in all three considered cases.

The action of $G$ and $G_{D}$ and hence of $K$ and $K_{D}$ translated into language of matrices is given by

$$
\begin{gathered}
U \cdot C_{v}=U C_{v} U^{t}, \text { bosons and fermions, } \\
(U, V) \cdot C_{v}=U C_{v} V^{t}, \text { distinguishable particles, }
\end{gathered}
$$

where ${ }^{t}$ denotes the transposition. Unless there is a danger of confusion we will let $\mathcal{H}$ denote any of the vector spaces $(1)$ and by $M=\mathbb{P}(\mathcal{H})$ the associated complex projective space. There are of course differences between these three cases, but conceptually speaking, these are slight. In order to facilitate a simultaneous treatment we let $n=N$ in the case of $S^{2} V$ and $2 n=N$ (resp. $2 n+1=N$ ) in the case of $\Lambda^{2} V$ where $N$ is even (resp. odd). Finally we will denote

$$
\begin{gathered}
s_{N}=e_{1} \vee e_{1}+e_{2} \vee e_{2}+\ldots+e_{N} \vee e_{N}, \\
a_{N}=e_{1} \wedge e_{2}+e_{3} \wedge e_{4}+\ldots+e_{2 n-1} \wedge e_{2 n}, \\
d_{N}=e_{1} \otimes e_{1}+e_{2} \otimes e_{2}+\ldots+e_{N} \otimes e_{N} .
\end{gathered}
$$

We will use $x_{N}$ to denote any of these points when treating the corresponding case. Notice that the matrices for these tensors are $C_{s_{N}}=2 I, C_{d_{N}}=I$, and $C_{a_{N}}=J$ where $J$ is a block diagonal matrix each $2 \times 2$ block being standard symplectic matrix, i.e.,

$$
J=\left(\begin{array}{cc}
0 & 1 \\
-1 & 0
\end{array}\right) \dot{+} \ldots \dot{+}\left(\begin{array}{cc}
0 & 1 \\
-1 & 0
\end{array}\right) \text {. }
$$

\section{The spherical property}

A complex Lie group $G$ of matrices is said to be reductive if it is the complexification $G=K^{\mathbb{C}}$ of its maximal compact subgroup. In the case of interest here $G=\mathrm{SL}_{N}(\mathbb{C})$ is the complexification of the compact subgroup $K=\mathrm{SU}(N)$. Let $H$ be an algebraic subgroup of $G$ and denote by $\Omega:=G / H$ corresponding homogenous space.

Definition 1. A G-homogenous spece $\Omega=G / H$ is said to be a spherical homogenous space if and only if some (and therefore every) Borel subgroup $B \subset G$ has an open dense orbit in $\Omega$.

Recall that by definition a Borel subgroup is a maximal connected solvable subgroup of $G$. In our particular case of interest where $G=\mathrm{SL}_{N}(\mathbb{C})$, the group of uppertriangular matrices, which can be regarded as the stabilizer of the standard full flag

$$
0 \subset \operatorname{Span}\left\{e_{1}\right\} \subset \operatorname{Span}\left\{e_{1}, e_{2}\right\} \subset \ldots \subset \operatorname{Span}\left\{e_{1}, \ldots e_{N-1}\right\} \subset \mathcal{H},
$$

is a good example of a Borel subgroup. In general, every two Borel subgroups are conjugate by an element of $G$. Hence in our case $B$ is a Borel subgroup if and only if it is the stabilizer of some full flag.

Suppose now that $M$ is an irreducible algebraic variety (for example a complex projective space $\mathbb{P}(\mathcal{H})$ ) equipped with a $G$-action. 
Definition 2. If $G$ has an open (Zariski dense) orbit $\Omega=G / H$ in $M$ and $\Omega$ is a spherical homogenous space, then $M$ is said to be a spherical embedding of $\Omega$.

In the next sections we show that spherical homogenous spaces possess a number of crucial properties which we will use to analyze the geometry of entangled states. Let us start with presenting a basic example of such spaces, vis. affine symmetric spaces which will play an important role in the due course.

Affine symmetric spaces. Let $\mathfrak{g}$ be a complex semisimple Lie algebra, $\theta: \mathfrak{g} \rightarrow \mathfrak{g}$ a complex linear involution, i.e., a Lie algebra automorphism with $\theta^{2}=\operatorname{Id}$, and $\mathfrak{g}=$ $\mathfrak{h} \oplus \mathfrak{p}$ the decomposition of $\mathfrak{g}$ into $\theta$-eigenspaces. The fixed point algebra $\mathfrak{h}$, i.e., the subspace belonging to the eigenvalue +1 of $\theta$, defines a (reductive - see Appendix)) closed subgroup $H$ in $G$. The complex homogenous space $G / H$ is in this case an affine symmetric space. In the Appendix we prove

\section{Proposition 1. Affine symmetric spaces are spherical.}

Our goal now is to prove that $M=\mathbb{P}(\mathcal{H})$, where $\mathcal{H}$ is the Hilbert space for two fermions, bosons or distinguishable particles, is a spherical embedding of some open dense orbit $\Omega$ of $G$ action ${ }^{1}$. We will first prove

Proposition 2. The orbits $G . s_{N}, G . a_{N}, G_{D} . d_{N}$ are affine symmetric spaces.

Proof. The isotropy subgroups (stabilizers) of the points $s_{N}, a_{N}$, and $d_{N}$ under the corresponding actions of $G$ or $G_{D}$ are given by $G_{s_{N}}=\left\{T \in G: T M_{s_{N}} T^{t}=\right.$ $\left.M_{s_{N}}\right\}=\left\{T \in G: T T^{t}=I\right\}, G_{a_{N}}=\left\{T \in G: T M_{a_{N}} T^{t}=M_{a_{N}}\right\}=\{T \in$ $\left.G: T J T^{t}=J\right\}$, and $G_{D d_{N}}=\left\{(T, S) \in G \times G: T M_{d_{N}} S^{t}=M_{d_{N}}\right\}=\{(T, S) \in$ $\left.G \times G: T S^{t}=I\right\}$, where we use the explicit forms of the matrices $M_{s_{N}}, M_{a_{N}}$, and $M_{d_{N}}$ given at the end of Section 2. On the other hand they are given as fixed point sets of the holomorphic involutions $\theta(T)=\left(T^{t}\right)^{-1}, \theta(T)=J\left(T^{t}\right)^{-1} J^{-1}$, and $\theta(T, S)=\left(\left(S^{t}\right)^{-1},\left(T^{t}\right)^{-1}\right)$, respectively ${ }^{2}$ Hence in all three cases cases the orbits $G . s_{N}=G / G_{s_{N}}, G \cdot a_{N}=G / G_{a_{N}}$, and $G_{D} \cdot d_{N}=G_{D} / G_{D d_{N}}$ are affine symmetric spaces.

Proposition 3. The orbits G. $s_{N}, G . a_{N}, G_{D} . d_{N}$ are open Zariski dense in the appropriate $\mathbb{P}(\mathcal{H})$.

Proof. Treated as quadratic forms the points $s_{N}, a_{N}, d_{N}$ are of maximal rank. Since the rank of such a form is the only invariant in the sense that any two forms of the same rank are equivalent modulo the standard $\operatorname{GL}(\mathcal{H})$-action, it follows that $\operatorname{GL}(\mathcal{H}) \cdot x_{N}$ is dense and open in $\mathcal{H}$ and $\operatorname{SL}(\mathcal{H}) \cdot x_{N}=G \cdot x_{N}$ is open in $M=\mathbb{P}(\mathcal{H})$

Proposition 4. $M=\mathbb{P}(\mathcal{H})$, where $\mathcal{H}$ is the Hilbert space for two fermions, bosons or distinguishable particles is spherical embedding of $G . s_{N}, G . a_{N}, G_{D} . d_{N}$ respectively

Proof. Follows from the above propositions.

\footnotetext{
1 The respective $G$ actions were defined in Section 2

2 On the level of the algebras the corresponding involutions (denoted by the same letter $\theta$ ) read, respectively $\theta(X)=-X^{t}, \theta(X)=-J X^{t} J^{-1}$, and $\theta(X, Y)=\left(-X^{t},-Y^{t}\right)$
} 


\section{Moment map}

In the following we show that it is very useful to view spherical varieties from the standpoint of symplectic geometry. To this end we devote this section to the concept of the moment map (see [4] for more details). We discuss it in general as well as in the specific context of our problem. Finally we derive an explicit formula for the moment map associated to the unitary action on the complex projective space.

4.1. General setting. Let $K$ be any connected, semisimple Lie group acting smoothly as group of symplectomorphisms of a symplectic manifold $(M, \omega)$, i.e.

$$
K \times M \ni(g, x) \mapsto \Phi_{g}(x) \in M, \quad \Phi_{g}^{*} \omega=\omega .
$$

For any $\xi \in \mathfrak{k}$ we define a fundamental vector field $\hat{\xi}$ for the $\Phi$ action

$$
\hat{\xi}(x)=\left.\frac{d}{d t}\right|_{t=0} \Phi_{\exp t \xi}(x) .
$$

In the following, to shorten the notation we will use $g x$ or $g . x$ instead of $\Phi_{g}(x)$ for $g \in G, x \in K$.

The map

$$
\widehat{\imath}:(\mathfrak{k},[\cdot, \cdot]) \rightarrow(\chi(M),[\cdot, \cdot]), \quad \xi \mapsto \hat{\xi},
$$

is a homomorphism of the Lie algebra $\mathfrak{k}$ into the Lie algebra $\chi(M)$ of the vector fields on $M$.

A vector field $X$ is said to be Hamiltonian if the Lie derivative of $\omega$ along it vanishes,

$$
X \in \operatorname{Ham}(M) \equiv \mathcal{L}_{H} \omega=0 .
$$

¿From the Cartan formula

$$
\mathcal{L}_{X} \omega=i_{X} \circ d \omega+d \circ i_{X} \omega .
$$

and $d \omega=0$ it follows that $d \circ i_{X} \omega=d \omega(X, \cdot)=0$ for a Hamiltonian vector field $X$, i.e. the 1-form $\omega(X, \cdot)$ is closed. If the first de Rham cohomology group of $M$ vanishes then all closed forms are exact, i.e., there exists a function $F$ such that $\omega(X, \cdot)=d F$. In this case the map $F \mapsto X_{F}$ which which assigns a Hamiltonian vector field $X_{F} \in$ $\operatorname{Ham}(M)$ to any smooth function $F \in C^{\infty}(M)$ via $\omega\left(X_{F}, \cdot\right)=d F$ is surjective. Moreover, one checks easily that if we define the Poisson bracket on $M$ in the usual way,

$$
\{F, G\}=\omega\left(X_{F}, X_{G}\right), \quad F, G \in C^{\infty}(M),
$$

the map

$$
\rho:\left(C^{\infty}(M),\{\cdot, \cdot\}\right) \rightarrow(\operatorname{Ham}(M),[\cdot, \cdot]), \quad F \mapsto X_{F},
$$

is a homomorphism of Lie algebras.

Observe now that since the action of $K$ on $M$ is symplectic we have $\mathcal{L}_{\hat{\xi}} \omega=0$ for the fundamental vector fields (4), i.e., the fundamental vector fields are Hamiltonian. 
Hence, under the assumption that the first de Rham cohomology group of $M$ vanishes, for each Lie algebra element $\xi$ there exists a function $\mu_{\xi}$ such that

$$
d \mu_{\xi}=\omega(\hat{\xi}, \cdot) .
$$

where $\xi$ is given by (4). Notice that for any $\xi$ the function $\mu_{\xi}$ is given only up to an arbitrary constant function. Since $\mathfrak{k}$ is finite dimensional, $\mu_{\xi}$ can be chosen to be linear in $\xi$, i.e.,

$$
\mu_{\xi}(x)=\langle\mu(x), \xi\rangle, \quad \mu(x) \in \mathfrak{k}^{*},
$$

where $\langle$,$\rangle is the pairing between \mathfrak{k}$ and $\mathfrak{k}^{*}$. In this way we obtain a map $\mu: M \rightarrow \mathfrak{k}^{*}$ called the moment map.

Without spoiling the properties of the moment map which we have just derived we still have the freedom to add a constant function to $\mu_{\xi}$ which is equivalent to adding a linear functional $\nu \in \mathfrak{k}^{*}$ to the mapping $\bar{\mu}$ from the Lie algebra $\mathfrak{k}$ to the space of smooth functions

$$
\mathfrak{k} \ni \xi \mapsto \bar{\mu}(\xi):=\mu_{\xi} \in C^{\infty}(M) .
$$

It can be shown that under a particular assumption about the group $K$ which is always fulfilled through the paper, vis., its semisimplicity, we can use this freedom to make $\bar{\mu}^{\prime}=\bar{\mu}+\nu$ a homomorphism of the Lie algebras $\mathfrak{k}$ and $C^{\infty}(M)$, the latter again endowed with the Lie algebra structure with the help of the Poisson bracket (8). In fact

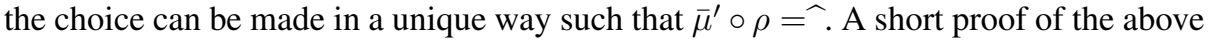
statement can be found in [4]. Here we remark only that if the second cohomology group of the Lie algebra $\mathfrak{k}$ vanishes, $H^{2}(\mathfrak{k}, \mathbb{R})=0$, then a $\nu$ making $\bar{\mu}$ the homomorphism always exists and if the first cohomology group vanishes as well, $H^{1}(\mathfrak{k}, \mathbb{R})=0$, then the choice of such $\nu$, and consequently of $\bar{\mu}^{\prime}$, is unique. The structure of cohomology groups of semisimple Lie algebras is characterized by Whitehead's lemmas, which state that, in particular for semisimple algebras, $H^{1}(\mathfrak{k}, \mathbb{R})=0=H^{2}(\mathfrak{k}, \mathbb{R})$. In the following we assume that our moment map $\mu$ is such that $\bar{\mu}$ is the above described unique homomorphism.

The group $K$ acts also on its Lie algebra $\mathfrak{k}$ via the adjoint action $\operatorname{Ad}_{g} \xi=g \xi g^{-1}$. The dual of the adjoint action is the coadjoint action on $\mathfrak{k}^{*}$,

$$
\left\langle\operatorname{Ad}_{g}^{*} \alpha, \xi\right\rangle=\left\langle\alpha, \operatorname{Ad}_{g^{-1}} \xi\right\rangle=\left\langle\alpha, g^{-1} \xi g\right\rangle,
$$

for $g \in K, \xi \in \mathfrak{k}$, and $\alpha \in \mathfrak{k}^{*}$. Since $\bar{\mu}$ is the unique homomorphism the moment map is equivariant (for details, consult again [4]), i.e., for each $x \in M$ and $g \in K$,

$$
\mu\left(\Phi_{g}(x)\right)=\operatorname{Ad}_{g}^{*} \mu(x)
$$

On each coadjoint orbit, $\Omega_{\alpha}=\left\{\operatorname{Ad}_{g}^{*} \alpha: g \in G\right\}$, there is a canonical symplectic structure - the so called Kirillov-Kostant-Souriau form given by

$$
\tilde{\omega}_{\alpha}(\tilde{\xi}, \tilde{\eta})=\langle\alpha,[\xi, \eta]\rangle,
$$

where $\tilde{\xi}, \tilde{\eta}$ are the fundamental vector fields associated to $\xi, \eta$ by the coadjoint action. Finally let us notice that using $[10]$ we get

$$
d \mu_{\xi}(x)(\widehat{\eta})=\omega(x)(\widehat{\xi}, \widehat{\eta}),
$$

and 
Proposition 5. For every $x \in M$ it follows that

$$
\operatorname{Ker}(d \mu(x))=\left(T_{x} K \cdot x\right)^{\perp_{\omega}},
$$

where by ${ }^{\perp_{\omega}}$ we denote the $\omega$-orthogonal complement

Let us denote by $\mathcal{F}_{x}:=\mu^{-1}(\mu(x))$ the $\mu$ fiber over $x \in M$. In general little can be said about the position of $\mathcal{F}_{x}$ with respect to $K . x$. Even if it is smooth we have only $T_{x} \mathcal{F}_{x} \subset\left(T_{x} K . x\right)^{\perp_{\omega}}$ which implies that $\mathcal{F}_{x}$ may not be entirely contained in the orbit $K . x$. However, when $K . x$ is coisotropic we have

Corollary 1. If K.x is co-isotropic, i.e., if $\left(T_{x} K . x\right)^{\perp_{\omega}} \subset T_{x} K . x$, the connected component of the $\mu$-fiber at $x$ is contained in K.x.

In order to emphasize the fact that the $\mu$-fiber might not be contained in $K . x$ let us consider following example.

Example 1. Let $\mathcal{H}_{1}=\mathbb{C}^{2}$, and $\mathcal{H}=\mathcal{H}_{1}^{\otimes 3}, K=S U_{2}, G=S L_{2}(\mathbb{C})^{\times 3}$. Denote by $\left\{e_{1}, e_{2}\right\} \subset \mathcal{H}_{1}$ the orthogonal basis of $\mathcal{H}_{1}$. Consider now two states

$$
\begin{gathered}
x_{1}=\sqrt{\frac{2}{3}} e_{1} \otimes e_{1} \otimes e_{1}+\frac{1}{\sqrt{3}} e_{2} \otimes e_{2} \otimes e_{2}, \\
x_{2}=\frac{1}{\sqrt{3}}\left(e_{2} \otimes e_{1} \otimes e_{1}+e_{1} \otimes e_{2} \otimes e_{1}+e_{1} \otimes e_{1} \otimes e_{2}\right) .
\end{gathered}
$$

Using formula 187 (see below) one easily checks that $\mu\left(x_{1}\right)=\mu\left(x_{2}\right)$. Notice however that the orbits $K . x_{1}$ and $K . x_{2}$ are disjoint as the orbits $G . x_{1}$ and $G . x_{2}$ are known to be disjoint [6].

4.2. Moment map on a complex projective space. Let us now restrict to a setting of special importance for our purposes, namely $M=\mathbb{P}(\mathcal{H})$. For this let $K$ be a connected compact Lie group acting linearly via a unitary representation on a Hilbert space $\mathcal{H}$. Note that the complexification $G:=K^{\mathbb{C}}$ is holomorphically represented on $\mathcal{H}$. Our goal now is to derive formula for the moment map. To this end let $\rho:=\log \|\cdot\|^{2}$ denote the associated ( $K$-invariant) norm-squared function and consider the $(1,1)$-form on $\mathcal{H} \backslash\{0\}$

$$
\omega:=-\frac{i}{2} \partial \bar{\partial} \rho
$$

For concrete calculations it is sometimes convenient to use local complex coordinates $z_{k}=x_{k}+i y_{k}$,

$$
\begin{gathered}
\omega=-\frac{i}{2} \sum_{k, l}\left(\partial_{z_{k}} \partial_{\bar{z}_{l}} \rho\right) d z_{k} \wedge d \bar{z}_{l}, \\
\partial_{z_{k}}=\frac{1}{2}\left(\partial_{x_{k}}-i \partial_{y_{k}}\right), \quad \partial_{\bar{z}_{k}}=\frac{1}{2}\left(\partial_{x_{k}}-i \partial_{y_{k}}\right), \\
d z_{k}=d x_{k}+i d y_{k}, \quad d \bar{z}_{k}=d x_{k}-i d y_{k} .
\end{gathered}
$$

The form $\omega$ is degenerate along the fibers of the standard projection $\mathcal{H} \backslash\{0\} \rightarrow$ $\mathbb{P}(\mathcal{H})$ and induces a symplectic form $\omega_{F S}$ on $\mathbb{P}(\mathcal{H})$ called the Fubini-Study structure 
associated to the given unitary structure on $\mathcal{H}^{3}$ We wish to define a moment map for the symplectic $K$-action on $\left(\mathbb{P}(\mathcal{H}), \omega_{F S}\right)$. For this we note that in general if $U$ is a Lie group containing $K$ and having a moment map $\mu_{U}: M \rightarrow \mathfrak{u}^{*}$ and $\pi: \mathfrak{u}^{*} \rightarrow \mathfrak{k}^{*}$ is the projection which is induced by the inclusion $\mathfrak{k} \hookrightarrow \mathfrak{g}$, then $\mu_{K}:=\pi \circ \mu_{U}$ is a moment map for the $K$-action. We now apply this to the case where $K$ is acting via a unitary representation as above and $U=U(\mathcal{H})$ is the full unitary group on $\mathcal{H}$.

The moment map for the action of the full unitary group can be obtained in the following way. For $\xi \in \mathfrak{u}(\mathcal{H})$ define

$$
\mu_{\xi}:=\frac{1}{4} J \widehat{\xi} \log \|\cdot\|^{2}=\frac{1}{4} J \widehat{\xi} \rho
$$

where $J$ is the linear complex structure on $\mathcal{H}$ regarded as a real Hilbert space. It is the matter of straightforward but tedious calculations to show that in fact,

$$
\omega:=-\frac{i}{2} \partial \bar{\partial} \rho=\frac{1}{4} d d^{c} \rho,
$$

where $d^{c} f(v)=(J v)(f)=d f(J v)$ for a tangent vector $v \in \mathcal{H}$. By these definitions we immediately get that $\mu: \mathcal{H} \rightarrow \mathfrak{u}(\mathcal{H})^{*}$ has the properties of a moment map, i.e., $d \mu_{\xi}=\omega(\hat{\xi}, \cdot)$ and $\mu(g(v))=\operatorname{Ad}_{g}^{*}(\mu(v))$. Since $\omega$ is degenerate and factors through $\mathcal{H} \backslash\{0\} \rightarrow \mathbb{P}(\mathcal{H})$, i.e., it is constant along the fibers of this projection, one shows easily that $\mu$ enjoys the same properties and defines a moment map $\mu: \mathbb{P}(\mathcal{H}) \rightarrow \mathfrak{u}(\mathcal{H})^{*}$ with respect to the $U(\mathcal{H})$-invariant Fubini-Study form $\omega_{F S}$. Let us explicitly compute this map. For $\xi \in \mathfrak{u}(\mathcal{H})$ from $(17)$ and the properties of the scalar product $\langle\cdot \mid \cdot\rangle$ we obtain

$$
\mu_{\xi}(v)=\frac{1}{4} J \widehat{\xi} \rho(v)=\left.\frac{1}{4} \frac{d}{d t}\right|_{t=0} \log \left\langle e^{i t \xi} v \mid e^{i t \xi} v\right\rangle=\frac{1}{2} \operatorname{Im} \frac{\langle v \mid \xi v\rangle}{\langle v \mid v\rangle}=\frac{i}{2} \frac{\langle v \mid \xi v\rangle}{\langle v \mid v\rangle} .
$$

Direct calculations with the help of (18) leads to yet another very useful formula for $\omega$,

$$
\omega\left(\hat{\xi}_{1}, \hat{\xi}_{2}\right)=-\frac{i\left\langle v \mid\left[\xi_{1}, \xi_{2}\right] v\right\rangle}{2\langle v \mid v\rangle}, \quad \xi_{1}, \xi_{2} \in \mathfrak{u}(\mathcal{H}) .
$$

Using 18 19, one easily checks that indeed both $\omega$ and $\mu$ factor through $\mathcal{H} \backslash\{0\} \rightarrow$ $\mathbb{P}(\mathcal{H})$.

The only missing ingredient in the formula $(18)$ is an explicit form for the Hermitian structure on $\mathcal{H}$ treated as space of (symmetric, antisymmetric, or general) matrices. To avoid computing we use the irreducibility of the representations of the considered groups on the corresponding $\mathcal{H}$ in all three cases. The arguments are slightly different for the diagonal action of $G=\mathrm{SL}_{\mathbb{C}}\left(\mathcal{H}_{1}\right)=\mathrm{SL}_{N}(\mathbb{C})$ in the symmetric and antisymmetric cases one one hand and the case of $G_{D}=\mathrm{SL}_{N}(\mathbb{C}) \times \mathrm{SL}_{N}(\mathbb{C})$ in the general case for distinguishable particles, so let us start with the former.

Proposition 6. The G-representations on $\mathcal{H}_{B}$ and $\mathcal{H}_{F}$ are irreducible.

Proof. Note that the action of the maximal complex torus $H$ is diagonalized in the bases chosen above for the two cases, i.e., for $v_{i j}$ a basis element $h\left(v_{i j}\right)=\chi_{i j}(h) v_{i j}$ for an explicitly known character $\chi_{i j}$. One checks that no two such characters are the same, i.e., in both cases the $H$-action is multiplicity-free. Now if $B$ is the Borel group

\footnotetext{
3 Let us recall that the Hermitian structure on $\mathcal{H}$ endows $\mathbb{P}(\mathcal{H})$ with a real Riemannian structure - the Fubini-Study metric. Together with $\omega$ it makes $\mathbb{P}(\mathcal{H})$ a Kähler manifold
} 
of upper-triangular matrices which contains $H$, then a $B$-eigenvector is certainly an $H$ eigenvector. But one checks that among the $v_{i j}$ the only $B$-eigenvector is $e_{1} \wedge e_{2}$ (resp. $\left.e_{1} \vee e_{1}\right)$ for $\mathcal{H}_{B}\left(\operatorname{resp} . \mathcal{H}_{F}\right)$.

Now recall that a holomorphic $G$-representation on a complex vector space $\mathcal{H}$ is irreducible if and only if some (and therefore every) Borel subgroup $B$ has exactly one fixed point in $\mathbb{P}(\mathcal{H})$, i.e., up to complex multiples there is a unique highest weight vector. Since this is the case in our examples, the desired result has been proved.

The following is the metric version of the criterion which was used above for the irreducibility of a representation.

Proposition 7. A representation of a compact Lie group $K$ as a group of complex linear transformations on a complex vectors space $\mathcal{H}$ is irreducible if and only if there is a unique (up to scalar factors) $K$-invariant unitary structure on $\mathcal{H}$.

As a consequence any $K$-invariant Hermitian structure which we choose on $\mathcal{H}$ will automorphically be the unique one which will define $\omega_{F S}$ and the associated moment map on $\mathbb{P}(\mathcal{H})$. Let us now return to regarding a point in $\mathcal{H}$ as a matrix $M$ and let

$$
\left\langle C_{1}, C_{2}\right\rangle:=\operatorname{Tr}\left(C_{1}^{\dagger} C_{2}\right)
$$

Corollary 2. Up to scalar multiples the Hermitian structure $\langle$,$\rangle is the unique K$ invariant structure on $\mathcal{H}=\mathcal{H}_{B}, \mathcal{H}_{F}$

Using this we get in both cases the following expression for the moment map

$$
\mu_{\xi}([v])=\frac{i}{2} \frac{\operatorname{Tr}\left(C_{v}^{\dagger} C_{v} \xi\right)}{\operatorname{Tr}\left(C_{v}^{\dagger} C_{v}\right)}
$$

In the case of distinguishable particles, i.e., for $\mathcal{H}=\mathcal{H}_{D} \cong \mathbb{C}^{N} \otimes \mathbb{C}^{N}$ we define a $K$-equivariant antilinear isomorphism $v \mapsto\langle v \mid\rangle$ on the second factor. This defines a symplectomorphism $\mathbb{C}^{N} \otimes \mathbb{C}^{N} \rightarrow \mathbb{C}^{N} \otimes\left(\mathbb{C}^{N}\right)^{*}$ from the standard symmplectic structure on the tensor product to the symplectic structure on $\operatorname{End}\left(\mathbb{C}^{N}\right)$ defined by the unitary pairing

$$
\left\langle C_{1}, C_{2}\right\rangle:=\frac{1}{2} \operatorname{Tr}\left(C_{1}^{\dagger} C_{2}\right)
$$

This identification is not complex linear, in fact the $G$-representation is not equivalent to the original one. However, since we are only interested in the symplectic phenomena related to the $K$-representation, this is of no importance.

As a result of the above we have reduced the problem to considering the $G$-representation on $W:=\operatorname{End}\left(\mathbb{C}^{N}\right)$ defined by $(A, B)(C):=A C B^{-1}$. Due to the fact that the $K$-representation on $W$ is irreducible, the above unitary structure is (up to a scalar factor) the unique $K$-invariant structure and the associated moment map is defined as before by $(20)$. 


\section{Brion's theorem}

In Section 4 we observed that the $\mu$-fiber may not be entirely contained in the $K$ orbit. Equivalently the moment map $\mu: M \rightarrow \mathfrak{k}^{*}$ may not separate all $K$ orbits. Continuing our exposition of symplectic aspects of spherical varieties we will give in this section conditions ensuring that $\mu$ does separate all $K$-orbits. This beautiful characterization is due to Brion [5]. Here we give a brief exposition based on [7].

Theorem 1. (Brion) Let $K$ be a connected compact Lie group acting on connected compact Kähler manifold $(M, \omega)$ by a Hamiltonian action and let $G=K^{\mathbb{C}}$. The following are equivalent

1. $M$ is a spherical embedding of the open $G$-orbit.

2. For every $x \in M$ the $\mu$-fiber $\mu^{-1}(\mu(x))$ is contained in K.x.

Let us elucidate again that 2 . states exactly that the moment map separates all $K$ orbits, i.e., from $\mu(x)=\mu(y)$ it follows $y \in K . x$. In this situation the local unitary equivalence of states $x$ and $y$ can be effectively checked by establishing whether $\mu(x)$ nad $\mu(y)$ lie on the same coadjoint orbit of $K$. This in turn can be easily seen by finding points where coadjoint orbits through $\mu(x)$ and $\mu(y)$ cross the dual to the maximal commutative subalgebra of $\mathfrak{k}$ (see [2]), or in simple words by diagonalizing $\mu(x)$ and $\mu(y)$ by the coadjoint action of $K$ and comparing the spectra which should coincide if both $\mu(x)$ and $\mu(y)$ belong to the same coadjoint orbit.

The Brion's theorem is one of the basic tools we use in our reasoning, hence we believe it is expedient to sketch its proof.

For the purpose of this paper it is important to understand the proof of Brion's theorem only in one direction, namely that 1 . implies 2. Following [7] there are two crucial ingredients in this proof. The first one is the so called Marsden-Weinstein reduction which we now briefly describe.

Let $\left(M, \omega_{M}\right)$ and $\left(N, \omega_{N}\right)$ be two symplectic manifolds equipped with a Hamiltonian action of a compact connected Lie group $K$. Let $\mu_{M}: M \rightarrow \mathfrak{k}^{*}$ and $\mu_{N}: N \rightarrow \mathfrak{k}^{*}$ be the corresponding moment maps. Notice first that $(M \times N, \omega)$, where

$$
\omega=\pi_{M}^{*} \omega-\pi_{N}^{*} \omega,
$$

and $\pi_{M}: M \times N \rightarrow M, \pi_{N}: M \times N \rightarrow N$ are natural projections, is a symplectic manifold. Indeed, the 2 -form $\omega$ is closed since $d$ commutes with $\pi_{N, M}^{*}$. It is also nondegenerate since assuming to the contrary that there is a vector $u=\left(u_{1}, u_{2}\right)$ such that for any $v=\left(v_{1}, v_{2}\right), \omega(u, v)=0$, implies that

$$
\omega(u, v)=\omega_{M}\left(u_{1}, v_{1}\right)-\omega_{N}\left(u_{2}, v_{2}\right)=0 .
$$

for any $v_{1}$ and $v_{2}$. Choosing $v_{1}=u_{1}$ and using nondegeneracy of $\omega_{N}$ we get $u_{2}=0$ and hence by nondegeneracy of $\omega_{1}$ we have $u_{1}=0$. It is easy to see now that the diagonal $G$ action on $M \times N$ is Hamiltonian with the moment map $\mu_{M \times N}: M \times N \rightarrow \mathfrak{k}$ given by

$$
\mu_{M \times N}=\mu_{M}-\mu_{N}
$$

Now let us apply this construction to $N=\Omega_{l}$ - the coadjoint orbit of $K$ through $l \in \mathfrak{k}$. Of course, in this particular setting

$$
\mu_{M \times \Omega_{l}}(x, k)=\mu_{M}-k, \quad k \in \Omega_{l},
$$


and the fiber of $\mu_{M \times \Omega_{l}}^{-1}(0)$ is given by

$$
\mu_{M \times \Omega_{l}}^{-1}(0)=\left\{(x, k): \mu_{M}(x)=k, k \in \Omega_{l}\right\} .
$$

It is easy to see now that

$$
\mu_{M \times \Omega_{l}}^{-1}(0) / K \cong \mu_{M}^{-1}(l) / \operatorname{Stab}(l)
$$

where $\operatorname{Stab}(l)=\left\{g \in K: \operatorname{Ad}_{g}^{*} l=l\right\}$.

The following lemma is the second key ingredient

Lemma 1. (Kirwan [8]) Let $K$ be a connected compact Lie group acting on connected compact Kähler manifold $(M, \omega)$ by a Hamiltonian action and let $\mu: M \rightarrow \mathfrak{k}^{*}$ be the moment map. Assume that $x, y \in \mu^{-1}(0)$ and $x \notin K . y$. Then there exists a $K^{\mathbb{C}}$ invariant open disjoint neighborhoods of $x$ and $y$ in $M$.

An obvious consequence of this lemma is

Corollary 3. Assume that $M$ is $K^{\mathbb{C}}$-almost homogenous, i.e. there is a point $x \in M$ such that $K^{\mathbb{C}} . x$ is open dense in $M$. Then $\mu^{-1}(0)$ is either empty or a single orbit of the $K$-action.

We are now ready to prove Theorem 1 . In order to do this we need to show that

$$
\mu_{M}^{-1}(M) / \operatorname{Stab}(l)
$$

is a single point for any $l \in \mu(M)$. But by the Marsden-Weinstein construction this is equivalent to showing that $\mu_{M \times \Omega_{l}}^{-1}(0) / K$ is a single point. In order to prove it is enough, by Corollary 3 to show that the diagonal action of $K^{\mathbb{C}}$ has an open dense orbit in $M \times \Omega_{l}$. Notice first that the coadjoint orbit is the homogenous space $\Omega_{l}=K^{\mathbb{C}} / P$ where $P=\operatorname{Stab}(l)$ is a parabolic subgroup. The isotropy subgroup of any point $k \in \Omega_{l}$ is parabolic group of the form $\operatorname{Stab}(k)=g P g^{-1}$ where $g . l=k$ and $g \in K^{\mathbb{C}}$. The orbit of diagonal action of $\operatorname{Stab}(k) \subset K^{\mathbb{C}}$ through $(x, k) \in M \times \Omega_{l}$ is $\left(\mathcal{O}_{x}, k\right)$. For each $k \in \Omega_{l}$ there is a Borel group $B \subset \operatorname{Stab}(k)$ and since $M$ is spherical for each $k \in \Omega_{l}$ there is $x_{k} \in M$ such that $\mathcal{O}_{x}$ is open (they are denoted by dashed lines in the figure 1). Now since $K^{\mathbb{C}}$ is acting transitively on $\Omega_{l}$ we get by the above reasoning that $\bigcup_{k \in \Omega_{l}} \mathcal{O}_{x_{k}}$ is open dense orbit of $K^{\mathbb{C}}$ diagonal action on $M \times \Omega_{l}$. Hence by Corollary 3 the quotient $\mu_{M \times \Omega_{l}}^{-1}(0) / K$ is a single point. 


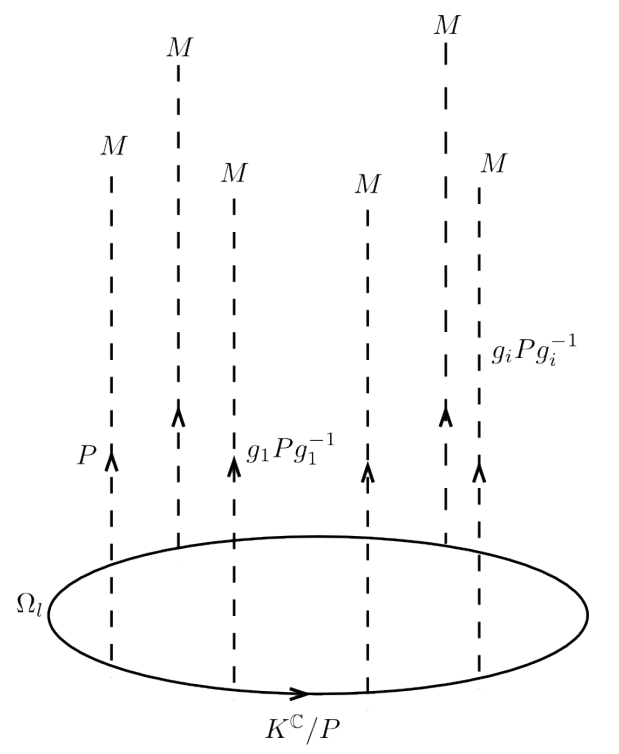

Fig. 1. The open dense orbit $\bigcup_{k \in \Omega_{l}} \mathcal{O}_{x_{k}}$ of $B$ in $M \times \Omega_{l}$

In order to emphasize the fact that it is not enough to assume that $M$ is $K^{\mathbb{C}}$-almost homogenous but we really need the spherical property in Brion's theorem let us consider the following example

Example 2. Let $\mathcal{H}_{1}=\mathbb{C}^{2}$, and $\mathcal{H}=\mathcal{H}_{1}^{\otimes 3}, K=S U_{2}, K^{\mathbb{C}}=S L_{2}(\mathbb{C})^{\times 3}$. Denote by $\left\{e_{1}, e_{2}\right\} \subset \mathcal{H}_{1}$ an orthogonal basis of $\mathcal{H}_{1}$. Consider a state

$$
x=\sqrt{\frac{2}{3}} e_{1} \otimes e_{1} \otimes e_{1}+\frac{1}{\sqrt{3}} e_{2} \otimes e_{2} \otimes e_{2} .
$$

It is the matter of direct calculations to show that $K^{\mathbb{C}} . x$ is open and dense in $\mathbb{P}(\mathcal{H})$ so $\mathbb{P}(\mathcal{H})$ is $K^{\mathbb{C}}$-almost homogenous. But from example 1 we know that $\mu$ does not separate all $K$ - orbits.

It should be also mentioned that in great generality it is known that every $\mu$-fiber is connected. In the case at hand, due to the fact that the coadjoint orbits $K . \mu(x)$ are simply-connected, this follows directly from Brion's theorem. Notice finally that combining Proposition 4 with Brion's theorem we get

Corollary 4. For $M=\mathbb{P}(\mathcal{H})$, where $\mathcal{H}$ is the Hilbert space for two fermions, bosons or distinguishable particles, the corresponding moment map $\mu: M \rightarrow \mathfrak{k}^{*}$ separates all K-orbits

\section{Generic $\mu$ - fibers}

Here, in all cases of interest we let $\Sigma$ be the closure of the $H$-orbit $H$. [ $\left.x_{N}\right]$, where $x_{N}=a_{N}, s_{N}, d_{N}$ in the projective space $M=\mathbb{P}(\mathcal{H})$. This is itself a complex and therefore symplectic linear projective subspace of $M$ which is invariant with respect to the action of the compact torus $T$. Therefore we have its moment map $\mu_{T}: \Sigma \rightarrow \mathfrak{t}^{*}$. 
Computation of $\mu$ along $\Sigma$. Let us begin by computing the restriction $\left.\mu\right|_{\Sigma}$. For this it is notationally simpler to compute $\mu$ as it is actually defined in the vector space $\mathcal{H}$. Observe that for any $v \in \mathcal{H}$ if $[v] \in \Sigma$, then $D_{v}=C_{v}^{\dagger} C_{v}$ is a non-negative diagonal matrix with some non-zero entry.

Proposition 8. If $[v] \in \Sigma$, then

$$
\mu_{\xi}([v])=\frac{i}{2} \frac{\operatorname{Tr}\left(C_{v}^{\dagger} C_{v} \xi\right)}{\operatorname{Tr}\left(C_{v}^{\dagger} C_{v}\right)}=\frac{i}{2} \frac{\operatorname{Tr}\left(D_{v} \xi\right)}{\operatorname{Tr}\left(D_{v}\right)} .
$$

At this point it will be expedient to abandon the parallel treatment of diagonal and non-diagonal action of $K$ and postpone the latter momentarily. Although the line of reasoning remains the same, the moment map is slightly different due to the fact that the Lie algebra $\mathfrak{k}=\mathfrak{s u}_{N}$ for the diagonal actions and $\mathfrak{k}=\mathfrak{s u}_{N} \oplus \mathfrak{s u}{ }_{N}$ in the general two-partite case and it is better to make the concrete calculations separately.

To treat the cases of diagonal action we decompose the Lie algebra $\mathfrak{s u}_{N}$ as $\mathfrak{t} \oplus \mathfrak{t}^{\perp}$ with respect to a (unique up to a constant factor) Ad-invariant trace-form. The elements of $\mathfrak{t}$ are diagonal of the form $i \operatorname{diag}\left(\varphi_{1}, \ldots, \varphi_{N}\right)$ with $\sum \varphi_{j}=0$. A basis of $\mathfrak{t}^{\perp}$ is given as follows: For $k<l$ let $E_{k l}$ be the matrix which is one in the i-th row and j-th column, i.e., the matrix corresponding to the tensor $e_{k} \otimes e_{l}$. The basis of $\mathfrak{t}^{\perp}$ is then given by the matrices $E_{k l}-E_{k l}^{t}$ and $i\left(E_{k l}+E_{k l}^{t}\right)$ with $k<l$. Using this basis one immediately observes the following fact (see [1] for detailed calculations)

Proposition 9. For $[v] \in \Sigma$ it follows that $\mu_{\xi}([v])=0$ for all $\xi \in \mathfrak{t}^{\perp}$.

If $[v] \in \Sigma$, for the description of $\mu_{\xi}$ with $\xi \in \mathfrak{t}$ it is useful to think about diagonal entries of $D_{v}$ as defining a probability measure $P([v])=\left(p_{1}([v]), \ldots, p_{N}([v])\right)$, where

$$
p_{j}([v])=\frac{\left(D_{v}\right)_{j j}}{\sum_{j=1}^{N}\left(D_{v}\right)_{j j}}, \quad \sum_{j=1}^{N} p_{j}([v])=1, \quad p_{i}([v]) \geq 0 .
$$

Proposition 10. If $[v] \in \Sigma$ and $\xi=i \operatorname{Diag}\left(\varphi_{1}, \ldots, \varphi_{N}\right)$, then

$$
\mu_{\xi}([v])=\sum p_{j}([v]) \varphi_{j} .
$$

We may thus regard $\left.\mu_{\xi}([v])\right)$ as the expected value of $\xi$ at $[v]$.

The restricted moment map. It is now convenient to identify $\mathfrak{k}^{*}$ with $\mathfrak{k}$. We do so by using the invariant bilinear form given by the trace and regard moment maps as having values in Lie algebras as opposed to their duals. To simplify the notation we regard a diagonal matrix $\xi=i \operatorname{diag}\left(\varphi_{1}, \ldots, \varphi_{N}\right) \in \mathfrak{t}$ as a vector $\left(\varphi_{1}, \ldots, \varphi_{N}\right)$. The following is then a translation of the above results.

Proposition 11. The restriction of the moment map $\mu: M \rightarrow \mathfrak{k}$ to $\Sigma$ is the T-moment map $\mu^{T}: \Sigma \rightarrow \mathfrak{t}$. This restricted moment map is given by the translated probability vector

$$
\mu([v])=\left(p_{1}([v]), \ldots, p_{N}([v])\right)-\frac{1}{N}(1, \ldots, 1) .
$$

Its fiber at $[v] \in \Sigma$ is the orbit $T$. $[v]$. Its image is the translated probability polyhedron

$$
P=\left\{\left(p_{1}-\frac{1}{N}, p_{2}-\frac{1}{N}, \ldots, p_{N}-\frac{1}{N}\right): p_{i} \geq 0 \text { for all } i \text { and, } \sum p_{i}=1\right\} .
$$


We say that a point $[v] \in \Sigma$ is generic if $p_{1}([v])>\ldots>p_{N}([v])>0$ and let $\Sigma_{g e n}$ denote the set of such points. The following is a direct consequence of the definitions.

Proposition 12. The restricted moment map has constant rank $n$ on $\Sigma_{\text {gen }}$ and maps $\Sigma_{\text {gen }}$ onto the interior of the translated probability polyhedron $P$.

Orbits of points in $\mu(\boldsymbol{\Sigma})$. Here we let $[v] \in \Sigma$ and analyze the restriction $\left.\mu\right|_{K \text {. }[v] \text {. Recall }}$ that the $K$-action on $\mathfrak{k}$ is the adjoint representation which in our matrix setup is just the conjugation. Thus the $K$-isotropy subgroup at a point $\mu([v])$ is just its centralizer in $K$. We may therefore restrict our attention to the intersection with the standard fundamental domain of the Weyl-group.

$$
\mathfrak{t}^{+}:=\left\{\left(q_{1}, \ldots, q_{N}\right) ; q_{1} \geq \ldots \geq q_{N}, \sum q_{i}=0\right\} .
$$

For $\xi \in \mathfrak{t}$ the orbit $K . \xi$ is a flag manifold which depends only on the equalities among the $q_{i}$. If

$q_{1}=\ldots=q_{d_{1}}>q_{d_{1}+1}=\ldots=q_{d_{1}+d_{2}}>\ldots>q_{d_{1}+\ldots+d_{k-1}+1}=\ldots=q_{d_{1}+\ldots+d_{k}}$

then $K . \xi$ is the flag manifold denoted by $F\left(d_{1}, \ldots, d_{k}\right)$. In that case the isotropy group $K_{\xi}$ is the stabilizer of the standard flag

$$
0<\operatorname{Span}\left\{e_{1}, \ldots e_{d_{1}}\right\}<\operatorname{Span}\left\{e_{1}, \ldots, e_{d_{1}+d_{2}}\right\}<\operatorname{Span}\left\{e_{1}, \ldots, e_{d_{1}+\ldots+d_{k}}\right\}=V .
$$

The following transversality property will be essential for our computations.

Proposition 13. If $\xi \in \mathfrak{t}$, then $T_{\xi}(K . \xi) \cap \mathfrak{t}=\{0\}$.

Proof. This is direct consequence of the fact that adjoint $K$ action does not change the spectrum of antihermitian matrix. More concretely notice that $g . \xi=\operatorname{Ad}_{g} \xi=g \xi g^{-1}$. So the tangent space $T_{\xi}(K . \xi)$ is spanned by vectors $\left[\xi, \xi_{1}\right]$ where $\xi_{1} \in \mathfrak{k}$. Since $\xi \in \mathfrak{t}$, i.e., $\xi$ is a diagonal matrix, it is obvious that if $\left[\xi, \xi_{1}\right]$ is diagonal than it is equal 0 .

Isotropy groups and $\mu$-fibers at generic points. For $[v] \in \Sigma_{\text {gen }}$ we describe here the isotropy groups $K_{[v]}$ and $K_{\mu([v])}$. In both cases the $\mu$-image of $\Sigma_{g e n}$ is the interior of the translated probability polyhedron. In the case of bosons this is open in $\mathfrak{t}$ and thus $K . \mu([v])$ is the full flag manifold $F(1,1, \ldots, 1)$. Thus the isotropy group $K_{\mu([v])}$ is the maximal torus $T$. Since $\mu$ is equivariant $K_{[v]}$ is contained in $K_{\mu([v])}=T$. This is the finite group $\Gamma$ consisting of diagonal matrices $\operatorname{diag}( \pm 1, \ldots \pm 1)$ with unit determinant.

For fermions the orbit $K . \mu([v])$ is the flag manifold $F(2,4, \ldots, \ldots, 2 n-2,2 n)$ in the case where $N=2 n$ is even and $\mathcal{F}(2,4, \ldots, 2 n-2,2 n, 2 n+1)$ in the case where $N=2 n+1$ is odd. In each $2 \times 2$-block the isotropy group is $\mathrm{U}_{2}$. The determinant condition then implies that $K . \mu([v])=\mathrm{S}\left(\mathrm{U}_{2} \times \ldots \times \mathrm{U}_{2}\right)$ in the case where $N=2 n$ and $K_{\mu([M])}=S\left(U_{2} \times \ldots \times U_{2} \times U_{1}\right)$ with $N=2 n+1$. In both cases there are $n$ $U_{2}$-factors.

To compute the isotropy subgroup $K_{[v]}$ we therefore only need to compute the $\mathrm{U}_{2}$ isotropy on $e_{1} \wedge e_{2}$ which is simply $\mathrm{SU}(2)=\mathrm{USp}(2)$. Thus in both cases, up to possible finite intersections $K_{[v]}$ is the product $S^{1}\left(\mathrm{SU}_{2} \times \ldots \times \mathrm{SU}_{2}\right)$ of $n$-copies of $\mathrm{SU}_{2}$ with $S^{1}=\left(e^{i \phi}: \phi \in[0,2 \pi[)\right.$. Let us summarize these result. 
Proposition 14. Let $[v] \in \Sigma_{g e n}$. For bosons $K_{\mu([v])}$ is the maximal torus $T$ and $K_{[v]}$ is the finite group $\Gamma:=\{\operatorname{Diag}( \pm 1, \ldots, \pm 1) \in K\}$. For fermions, if $N=2 n$ is even,

$$
K_{\mu([v])}=\mathrm{S}(\mathrm{U}(2) \times \ldots \times \mathrm{U}(2))
$$

and $K_{[v]}$ is the product $\mathrm{SU}_{2} \times \ldots \times \mathrm{SU}_{2}$ of $n$ copies of $\mathrm{SU}_{2}$. If $N=2 n+1$, then, modulo the determinant condition,

$$
K_{\mu([v])}=\mathrm{S}\left(\mathrm{U}_{2} \times \ldots \times \mathrm{U}_{2} \times \mathrm{U}_{1}\right)
$$

is the product of $n$ copies of $\mathrm{U}_{2}$ with $\mathrm{U}_{1}$. In that case

$$
K_{[v]}=S^{1}\left(\mathrm{SU}_{2} \times \ldots \times \mathrm{SU}_{2}\right) .
$$

As a result it is a simple matter to describe the fiber $\mathcal{F}_{[v]}=\mu^{-1}(\mu([v]))$ of the homogeneous fibration $K_{[v]} \rightarrow K_{\mu([v])}$ which by Corollary 4 is the $\mu$-fiber at $[v]$.

Proposition 15. For bosons if $[v] \in \Sigma_{\text {gen }}$, the $\mu$-fiber $\mathcal{F}_{[v]}$ can be identified with the maximal torus $T$ modulo the finite group $\Gamma$. In the case of fermions it is the $(n-1)$ dimensional subtorus

$$
T_{1}=\left\{\operatorname{diag}\left(\lambda_{1}, \lambda_{1}, \ldots, \lambda_{n}, \lambda_{n}\right) \in \mathrm{SU}_{N}\right\} .
$$

It is in fact not a coincidence that in the case of spherical action the generic fiber of the moment map is a torus of dimension complementary to that of the generic $K$-orbit (see [7] for this and other results for such an actions).

The slice property. Using the above results on the properties of the moment map along $\Sigma$, we will now show that $\Sigma$ has the so called slice property. Namely we have following general fact

Proposition 16. Let $K$ be a connected compact group acting smoothly on a connected real analytic manifold $M$ and $N$ be a closed analytic submanifold. Let $M_{\max }$ denote the open subset of points $x \in M$ so that K.x is of maximal dimension and assume that the complement of $M_{\max } \cap N$ is nowhere dense in $\Sigma$. Furthermore, suppose that if $x \in M_{\max } \cap N$, it follows that $T_{x} K . x+T_{x} N=T_{x} M$. Then $K . N=M$.

Proof. By assumption the complement $E$ of $M_{\max } \cap N$ is a nowhere dense analytic set. Local linearization of isotropy groups shows tht $E=K . E$ is at least 2-codimensional and therefore the open complement $M \backslash K . E$ is connected. The condition $T_{x} K . x+$ $T_{x} N=T_{x} M$ for all $x \in M_{\max } \cap N$ implies that $K .(N \backslash E)$ is open. Since the complement of $K . N$ is open and $M \backslash K . E$ is connected, the desired result follows

The closed analytic submanifold $N \subset M$ which has properties given in the Proposition 16 is called slice. It should be also remarked that Proposition 16 is not the most general of its type, but that it is sufficient for proving the slice property in our context.

Theorem 2. The complex submanifold $\Sigma:=\operatorname{cl}\left(H . x_{N}\right)$ has the slice property, i.e. $K . \Sigma=M$. 
Proof. By Proposition 16, it is enough to show the transversality condition is fulfilled for $[v] \in \Sigma_{g e n}$. For both fermions and bosons the $\mu$-fiber at $[v]$ is a torus $T_{0}$ which is contained in both $\Sigma$ and $K$. [v]. Furthermore, $\left.\mu\right|_{\Sigma_{g e n}}$ has constant rank with image in $\mathfrak{t}$ and $K . \mu([v])$ is transversal to $\mathrm{t}$. Thus

$$
\operatorname{dim}\left(T_{[v]} \Sigma+T_{[v]} K \cdot[v]\right)=\operatorname{dim} T_{[v]} K \cdot[v]+\operatorname{dim} T_{0}=\operatorname{dim} T_{[v]} M .
$$

Using this result it is now possible to give an exact parameterizations of the $K$-orbits in $M$. For this let $H_{\mathbb{R}}$ be the real points of $H$ defined as the subgroup corresponding to the Lie algebra $i$ t. If

$$
H_{\mathbb{R}}^{+}:=\left\{\operatorname{diag}\left(\lambda_{1}, \ldots, \lambda_{N}\right): \lambda_{1} \geq \ldots \geq \lambda_{N}>0\right\}
$$

and $\Sigma_{\mathbb{R}}^{+}:=\operatorname{cl}\left(H_{\mathbb{R}}^{+} \cdot x_{N}\right)$, then every $T$-orbit in $\Sigma$ intersects $\Sigma_{\mathbb{R}}^{+}$in a unique point. Furthermore, if $\left[v_{1}\right],\left[v_{2}\right] \in \Sigma_{\mathbb{R}}^{+}$, then $\mu\left(\left[v_{1}\right]\right)=\mu\left(\left[v_{2}\right]\right)$ if and only if $\left[v_{1}\right]=\left[v_{2}\right]$. Since the $K$-orbits in $\mu(M)$ are exactly parameterized by $\mu\left(\Sigma_{\mathbb{R}}^{+}\right)$, the following is immediate.

Corollary 5. Every $K$-orbit in $M$ intersects $\Sigma_{\mathbb{R}}^{+}$in exactly one point.

\section{Orbital symplectic structure}

Here, for an arbitrary point $[v] \in M$ we describe the orbit $K .[v]$ along with the partial symplectic structure which is a restriction of the symplectic structure $\omega$ of the projective space $M=\mathbb{P}(\mathcal{H})$. From Corollary 4 we know that the $\mu$-fiber $\mathcal{F}_{[v]}$ is just the fiber of the homogeneous fibration $K / K_{[v]} \rightarrow K / K_{\mu([v])}$. Thus, in order to describe $K$. $[v]$ and its induced partial symplectic struture, it is enough to compute the isotropy groups $K_{[v]}$ and $K_{\mu([v])}$, thereby describing the fiber $\mathcal{F}_{[v]}$. By Theorem 16 it is enough to do this for $[v] \in \Sigma_{\mathbb{R}}^{+}$.

For $[v] \in \Sigma_{\mathbb{R}}^{+}$the image $\mu([v])$ is the translated probability vector

$$
\mu([v])=\left(p_{1}([v]), \ldots, p_{N}([v])\right)-\frac{1}{N}(1, \ldots, 1)=\left(q_{1}, \ldots q_{N}\right) .
$$

Since $[v] \in \Sigma_{\mathbb{R}}^{+}$, it follows that $q_{1} \geq \ldots \geq q_{N} \geq 0$. We define the vector $d([v])=$ $\left(d_{1}, \ldots d_{k}\right)$ by the condition

$$
q_{1}=\ldots=q_{d_{1}}>q_{d_{1}+1}=\ldots=q_{d_{1}+d_{2}}>\ldots>q_{d_{1}+\ldots d_{k-1}+1}=\ldots=q_{d_{1}+\ldots d_{k}},
$$

where $d_{1}+\ldots+d_{k}=N$. The vector $d([v])$ is of course uniquely determined by $[v] \in \Sigma_{\mathbb{R}}^{+}$. For example, in the case of bosons $v=\lambda_{1} e_{1}^{2}+\ldots \lambda_{N} e_{N}^{2}$ with $\lambda_{1} \geq \ldots \geq$ $\lambda_{N} \geq 0$ define the multiplicity vector $d([v])$. In the case of fermions we are dealing with $v=\lambda_{1} e_{1} \wedge e_{2}+\lambda_{2} e_{3} \wedge e_{4}+\ldots+\lambda_{n} e_{2 n-1} \wedge e_{2 n}$ with $\lambda_{1} \geq \ldots \geq \lambda_{n} \geq 0$. If the equalities for the the $\lambda_{j}$ define $\left(\tilde{d}_{1}, \ldots, \tilde{d}_{k}\right)$, then $d_{j}=2 \tilde{d}_{j}$ for all $j$. Note that if $N=2 n+1$ and $\mu([v])=\left(q_{1}, \ldots, q_{N}\right)$ it is always the case that $q_{N}=0$ so that it is always the case $d_{k} \geq 1$.

Flag manifolds in the $\mu$-image. Let $d([v])=\left(d_{1}, \ldots, d_{k}\right)$ be the vector 22 determined by $[v] \in \Sigma_{\mathbb{R}}^{+}$.

Proposition 17. For $[v] \in \Sigma_{\mathbb{R}}^{+}$the orbit $K . \mu([v])$ is the flag manifold $F\left(d_{1}, \ldots, d_{k}\right)=$ $K / K_{\mu([v])}$ where

$$
K_{\mu([v])}=\mathrm{S}\left(\mathrm{U}_{d_{1}} \times \ldots \times \mathrm{U}_{d_{k}}\right)
$$

is the product of unitary groups with the condition of unit determinant.

In order to describe the $\mu$-fiber $\mathcal{F}_{[v]}$ we must only describe the isotropy group $K_{[v]}$. 
The $\mu$-fiber along $\boldsymbol{\Sigma}_{\mathbb{R}}^{+}$. To simplify the discussion, we disregard the finite groups which arise in the calculations, e.g., replacing orthogonal groups with special orthogonal groups. Let us first handle the case of bosons. Restricting to the region $\Sigma_{\mathbb{R}}^{+}$, we let

$$
v=\sqrt{q_{1}}\left(e_{1}^{2}+\ldots+e_{d_{1}}^{2}\right)+\ldots+\sqrt{q_{k}}\left(e_{d_{1}+\ldots+d_{k-1}}^{2}+\ldots+e_{N}^{2}\right),
$$

where $e_{i}^{2}=e_{i} \vee e_{i}$. There are two situations which arise. First, if $q_{k} \neq 0$, i.e., if the associated quadratic form is nondegenerate, then it is best to express

$$
K_{\mu([v])}=\left(S^{1}\right)^{k-1}\left(\mathrm{SU}_{d_{1}} \times \ldots \times \mathrm{SU}_{d_{k}}\right)
$$

Since $K_{[v]} \subset K_{\mu([v])}$, one obtains

$$
K_{[v]}=\mathrm{SO}_{d_{1}} \times \ldots \mathrm{SO}_{d_{k}},
$$

where $S O_{d_{i}}$ are real orthogonal groups. If $q_{k}=0$, then it is expedient to express

$$
K_{\mu([v])}=\left(S^{1}\right)^{k-2}\left(\mathrm{SU}_{d_{1}} \times \ldots \times \mathrm{SU}_{d_{k}-1}\right) \times \mathrm{U}_{d_{k}} .
$$

and

$$
K_{[v]}=\left(\mathrm{SO}_{d_{1}} \times \ldots \times \mathrm{SO}_{d_{k}-1}\right) \times \mathrm{U}_{d_{k}} .
$$

For a clean statement, as in our calculations above we replace the fiber $\mathcal{F}_{[v]}$ by a covering space $\tilde{\mathcal{F}}_{[v]}$. This differs from $\mathcal{F}_{[v]}$ by a finite group quotient which has been neglected above and if necessary can be easily computed. For the statement we denote the symmetric space $\mathrm{SU}_{m} / \mathrm{SO}_{m}$ by $S_{m}$.

Proposition 18. For bosons and $[v] \in \Sigma_{\mathbb{R}}^{+}$with multiplicity vector $d=d([v])$ where $v$ is nondegenerate

$$
\tilde{\mathcal{F}}_{[v]}=T_{k-1}\left(S_{d_{1}} \times \times S_{d_{k}}\right)
$$

where $T_{k-1}$ is a $(k-1)$-dimensional torus. If the tensor $v$ is degenerate, then

$$
\tilde{\mathcal{F}}_{[v]}=T_{k-2}\left(S_{d_{1}} \times \times S_{d_{k-1}}\right) .
$$

Now for fermions we choose the point

$$
v=\sqrt{q_{1}} E_{d_{1}}+\ldots+\sqrt{q_{k}} E_{d_{k}}
$$

where $E_{d_{j}}:=e_{d_{1}+\ldots+d_{j-1}+1} \wedge e_{d_{1}+\ldots+d_{j-1}+2}+\ldots+e_{d_{1}+\ldots+d_{j}-1} \wedge e_{d_{1}+\ldots+d_{j}}$. As above there are two situations which arise depending on whether or not the tensor $v$ is degenerate. Note that the case of $N=2 n+1$ is always degenerate. Using the same principles as were applied for the computation in the case of bosons, we compute $K_{[v]}$. For this we denote the symmetric space $\mathrm{SU}_{m} / \mathrm{USp}_{m}$ by $A_{m}$.

Proposition 19. For fermions if $[v] \in \Sigma_{\mathbb{R}}^{+}$has a multiplicity vector $d([v])=\left(d_{1}, \ldots, d_{k}\right)$, then in the case when $v$ is nondegenerate

$$
\tilde{\mathcal{F}}_{[v]}=T_{k-1}\left(A_{1} \times \ldots \times A_{d_{k}}\right) .
$$

In the case when $v$ is degenerate

$$
\tilde{\mathcal{F}}_{[v]}=T_{k-2}\left(A_{1} \times \ldots \times A_{d_{k-1}}\right) .
$$


Summary of results. Omitting the technical descriptions which are given above, we now summarize our main results.

Theorem 3. Every orbit $K .[v]$ in $M=\mathbb{P}(\mathcal{H})$ intersects $\Sigma_{\mathbb{R}}^{+}$in exactly one point. If $d([v])=\left(d_{1}, \ldots, d_{k}\right)$ is the associated multiplicity vector, then the image orbit $K . \mu([v])$ is the flag manifold $F\left(d_{1}, \ldots, d_{k}\right)$. The full image $\mu(M)$ is the union of the orbits $K . \xi$ where $\xi$ is in the translated probability polyhedron $P-\frac{1}{N}(1, \ldots, 1)$. The $\mu$-fiber $\mathcal{F}_{[v]}$ at $[v] \in M$ is the fiber of the homogeneous fibration $K / K_{[v]}^{N} \rightarrow K / K_{\mu([v]) \text {. }}$ Depending on the case and whether or not tensors $v$ is degenerate, its fiber is the product of a torus and certain symmetric spaces which are explicitly described above.

Recall that by Corollary 4

$$
\operatorname{dim} \mathcal{F}_{[v]}=D([v]),
$$

where $D([v])$ is the degeneracy of the canonical symplectic form on $M$ restricted to the orbit $K .[v]$. For precise computation of dimension of an orbit and the degeneracy of its induced canonical form it is only necessary to know the dimensions of the symmetric spaces. For completeness we therefore remark that

$$
\operatorname{dim} S_{m}=\left(m^{2}-1\right)-\frac{m(m+1)}{2}=\frac{(m+1)(m-2)}{2}
$$

and

$$
\operatorname{dim} A_{m}=\left(m^{2}-1\right)-\frac{m(m-1)}{2}=\frac{m^{2}+m-2}{2} .
$$

One should note that in both cases there is only one symplectic orbit, $K$. [ $\left.e_{1} \vee e_{1}\right]$ and $K$. $\left[e_{1} \wedge e_{2}\right]$ in the respective cases. This is the projectivization of the orbit of the highest weight vector and is of course complex. The only isotropic orbit is that which is described by $d=(1, \ldots, 1)$ with trivial degeneracy in the cases of bosons and fermions with $N=2 n$, and 1-dimensional degeneracy in the case of fermions with odd $N$.

\section{Distinguishable particles}

Let us now return to the case of distinguishable particles. As already mentioned the reasoning is similar up to slight details of concrete computations. In particular, it is prudent to consider the projectivization $\Sigma=H . d_{N}$ in $\mathbb{P}\left(\mathcal{H}_{D}\right)$ of the set of diagonal matrices in $\mathcal{H}$ as a potential thick slice which is perfectly aligned with respect to $\mu$. The first relevant observation cocerns $K . \Sigma$.

\section{Proposition 20.}

$$
K . \Sigma=\mathbb{P}\left(\mathcal{H}_{D}\right)
$$

Proof. Suppose $\operatorname{rank}\left(C_{v}\right)=n$ is maximal. Since we are dealing with the projective space $\mathbb{P}\left(\mathcal{H}_{D}\right)$, we may assume that $C_{v} \in \mathrm{SL}_{N}(\mathbb{C})$ and consider the $K$-orbit of $[v]$. It is well known that the projectivization of the set of matrices which have maximal rank is in $K . \Sigma$ as every matrix $C_{v} \in S L_{N}(\mathbb{C})$ can be diagonalized by two unitary matrices [9]. Since the set of states corresponding to maximal rank matrices $C_{v}$ is open and dense in $\mathcal{H}_{D}$ and $K . \Sigma$ is closed, the result follows. 
Now we carry out an argument which is completely analogous to the arguments in the case of bosons. The formula for the moment map is slightly different so let us sketch the calculation in a bit of detail. We choose the maximal toral algebra $\mathfrak{t}_{D}$ to be the direct sum $\mathfrak{t} \oplus \mathfrak{t}$ of the diagonal matrices in the Lie algebra $\mathfrak{k}_{D}=\mathfrak{k} \oplus \mathfrak{k}$. Just as before we note that the elements in $\left.\mu\right|_{\Sigma}$ annihilate the orthogonal complement $\mathfrak{t}^{\perp}$ of maximal toral algebra. After identifying $\mathfrak{k}^{*}$ with $\mathfrak{k}$ by the Ad-invariant scalar product given by trace this means

$$
\left.\mu\right|_{\Sigma}: \Sigma \rightarrow \mathfrak{t}_{D}
$$

If $v=\lambda_{1} e_{1} \otimes e_{1}+\ldots+\lambda_{N} e_{N} \otimes e_{N}$, then for $\xi=i\left(\varphi_{1}, \ldots, \varphi_{n}\right) \oplus i\left(\psi_{1}, \ldots, \psi_{n}\right) \in \mathfrak{t}$, it follows that

$$
\mu_{\xi}([v])=\sum p_{i}([v])\left(\varphi_{i}+\psi_{i}\right)
$$

where the probability distribution vector is given by $P([v])=\left(p_{1}([v]), \ldots, p_{N}([v])\right)$,

$$
p_{i}([v])=\frac{\left|\lambda_{i}\right|^{2}}{\sum_{j=1}^{N}\left|\lambda_{j}\right|^{2}} .
$$

In order to regard $\mu([v])$ as a vector in $\mathfrak{t}_{D}=\mathfrak{t} \oplus \mathfrak{t}$ we must translate it:

$$
\mu([v])=\left(P([v])-\frac{1}{N} I, P([v])-\frac{1}{N} I\right),
$$

where

$$
I=(1, \ldots, 1)
$$

The image $\mu(\Sigma)$ gives all translated finite probability distributions which can arise. A fundamental region of the Weyl group is given by the image $\mu\left(\Sigma^{+}\right)$where the elements of $\Sigma^{+}$are of the form

$$
v=\lambda_{1}\left(e_{1}^{2}+\ldots+e_{d_{1}}^{2}\right)+\ldots+\lambda_{k}\left(e_{d_{1}+\ldots+d_{k-1}+1}^{2}+\ldots+e_{d_{1}+\ldots+d_{k}}^{2}\right),
$$

where $\lambda_{j} \in \mathbb{R}$ for all $j$ and $\lambda_{1}>\lambda_{2}>\ldots>\lambda_{k}>0$ and $d_{j}$ denotes the dimension of the subspace where $v$ has eigenvalue $\lambda_{j}, e_{i}^{2}=e_{i} \otimes e_{i}$ and $\operatorname{rank}(v)=d_{1}+\ldots+d_{k}$.

To complete our analysis of this situation we must describe in detail the fibration $K / K_{[v]} \rightarrow K / K_{\mu([v])}$. Let us begin by computing the $K$-isotropy group at a generic point $v \in \Sigma_{\text {gen }}$, i.e., where $\lambda_{1}>\lambda_{2}>\ldots \lambda_{n}>0$. Using the fact that $C_{v}^{\dagger}=C_{v}$ one shows that $K_{[v]}$ is simply $\Delta\left(T_{D}\right)=T \times T^{-1}$ which acts by scalar multiplication.

The isotropy group $K_{\mu([v])}$ is in every case the centralizer of $\left(P([v])-\frac{1}{N} I, P([v])-\right.$ $\left.\frac{1}{N} I\right)$. Thus, in the generic case it is just the full maximal torus $T_{D}$. Consequently, the coadjoint orbit is $K / T_{D}$ and the fiber is the group manifold $T_{D} / \Delta\left(T_{D}\right)$.

In the general case where the elements of $v$ occur with multiplicities $d_{1}, \ldots, d_{k}$ and $v$ is not necessarily of maximal rank a similar calculation is made to show that the base $K / K_{\mu([v])}$ is the 2-fold product $F\left(d_{1}, \ldots, d_{k}\right) \times F\left(d_{1}, \ldots, d_{k}\right)$ of the corresponding flag manifold. In this case the fiber is the product of the group manifolds $\left(\mathrm{SU}_{d_{i}} \times\right.$ $\left.\mathrm{SU}_{d_{i}}\right) / \mathrm{SU}_{d_{i}}$. It is perhaps noteworthy that just as in the previous two cases these fibers are symmetric spaces. 


\section{Summary}

We presented the exact description of the partial symplectic structure of all $K$-orbits in $M=\mathbb{P}(\mathcal{H})$ for two bosons, fermions and distinguishable particles. To do this we first noticed that in all cases $M$ is in fact a spherical variety, i.e., it is the closure of an open dense orbit of the Borel group. This observation turns out to be very fruitful. Namely, by Brion's theorem ([5]) it implies that the moment map $\mu: M \rightarrow \mathfrak{k}^{*}$ parameterizes all $K$-orbits in $M$, i.e., $\mu$ maps the set of $K$-orbits in $M$ bijectively onto the set of $K$-orbits in its image. We gave the exact description of the fibers $\mathcal{F}_{x}=\mu^{-1}(\mu(x))$ of the moment map. Remarkably in all three cases these fibers (up to very simple finite-coverings) are products of certain symmetric spaces.

We believe that the notion of spherical variety should play an important role also in case of multipartite systems. Of course by simple dimensional arguments $M$ is typically not spherical in this case but it might happen that certain of $K^{\mathbb{C}}$-orbits (class of SLOCC states) enjoy this property. We postpone these problems to the forthcoming publications.

\section{Acknowledgments}

We gratefully acknowledge the support of SFB/TR12 Symmetries and Universality in Mesoscopic Systems program of the Deutsche Forschungsgemeischaft, ERC Grant QOOLAP, a grant of the Polish National Science Centre under the contract number DEC-2011/01/M/ST2/00379 and Polish MNiSW grant no. IP2011048471.

\section{Appendix}

Complex symmetric spaces. Let $G$ be a reductive complex Lie group, i.e., a connected complex Lie group which is the complexification of some (and therefore any) maximal compact subgroup $U$. At the Lie algebra level this means that $\mathfrak{g}$ is the direct sum $\mathfrak{u}+i \mathfrak{u}$. Semisimple complex Lie groups such as the classical groups $\mathrm{SL}_{n}(\mathbb{C}), \mathrm{SO}_{n}(\mathbb{C})$ and $\mathrm{Sp}_{2 n}(\mathbb{C})$ are the main examples which occur in our applications. Typical choices for the maximal compact subgroups $U$ in the classical groups are $\mathrm{SU}_{n}, \mathrm{SO}_{n}(\mathbb{R})$ and $\mathrm{USp}_{2 n}$. It is important to note that $G$ can be holomorphically embedded in some $\mathrm{SL}_{N}(\mathbb{C})$ so that $U$ is contained in $\mathrm{SU}_{N}$. Thus, $\mathfrak{g}=\mathfrak{u}+i \mathfrak{u}$ is such that the operators in $\mathfrak{u}$ are antiHermitian and those in $i \mathfrak{u}$ are Hermitian. The Lie algebra $\mathfrak{u}$ is a real form of $\mathfrak{g}$ in the sense that there there is an antilinear Lie algebra involution $\sigma: \mathfrak{g} \rightarrow \mathfrak{g}$ with $\mathfrak{u}=\operatorname{Fix}(\sigma)$. In this case $\sigma \mid \mathfrak{u}=\operatorname{Id}_{\mathfrak{u}}$ and $\sigma \mid i \mathfrak{u}=-\operatorname{Id}_{i \mathfrak{u}}$.

One often writes $\mathfrak{g}=\mathfrak{g}_{u}+\mathfrak{p}$ for the decomposition defined by $\sigma$. As was noted above, $\mathfrak{p}$ can be regarded as a subspace of the full space of Hermitian matrices in $\mathfrak{g l}_{N}(\mathbb{C})$ so that $\exp : \mathfrak{p} \cong P$ is a diffeomorphism onto a space of positive-definite Hermitian matrices. Furthermore, $G$ splits accordingly, i.e. $G=G_{u} P$ is a product so that $G / G_{u} \cong P$ is diffeomorphic to some $\mathbb{R}^{m}$. Since maximal compact subgroups of simple Lie groups are in fact maximal and any two are conjugate, it follows that the set $\mathcal{C}$ of maximal compact subgroups of $G$ can be identified with $G / G_{u}$ or equivalently with $P$.

Complex symmetric spaces which play a role in our investigations are by definition complex homogenous spaces $G / K$ where $K$ is the fixed point set of a holomorphic involution $\theta: G \rightarrow G$. Note that $\theta$ induces a diffeomorphism $\theta: \mathcal{C} \rightarrow \mathcal{C}$ and recall 
that no finite group of diffeomorphism of a cell can act freely. Therefore $\theta$ has a fixed point on $\mathcal{C}$ or equivalently $\theta$ stabilizes some compact real form which without loss of generality can be taken to be $G_{u}$. Let us consider this matter at the Lie algebra level where we decompose $\mathfrak{g}=\mathfrak{g}_{u}+i \mathfrak{g}_{u}$ into $\sigma$ eigenspaces. Since $\theta: \mathfrak{g} \rightarrow \mathfrak{g}$ is complex linear and $\theta$ stabilizes $\mathfrak{g}_{u}$, it is immediate that this splitting is $\theta$-invariant. Let $\mathfrak{g}_{u}=\mathfrak{g}_{u}^{+}+\mathfrak{g}_{u}^{-}$be the $\theta$-decomposition of $\mathfrak{g}_{u}$ and note that the $\theta$-decomposition of $i \mathfrak{g}_{u}$ is

$$
i \mathfrak{g}_{u}=\left(i \mathfrak{g}_{u}\right)^{+}+\left(i \mathfrak{g}_{u}\right)^{-}=i \mathfrak{g}_{u}^{+}+i \mathfrak{g}_{u}^{-} .
$$

Thus

$$
\mathfrak{g}=\mathfrak{g}^{+}+\mathfrak{g}^{-}=\left(\mathfrak{g}^{+} u+i \mathfrak{g}_{u}^{+}\right)+\left(\mathfrak{g}_{u}^{-}+i \mathfrak{g}_{u}^{-}\right) .
$$

is the $\theta$-splitting of $\mathfrak{g}$. In particular, since $\mathfrak{k}=\mathfrak{g}^{+}$, it follows that the fixed subgroup $K$ is the complexification of the compact subgroup $K_{0}$ which is associated to the Lie algebra $\mathfrak{g}_{u}^{+}$.

\section{Proposition 21. The $\theta$-fixed subgroup $K$ is reductive.}

Proof. Since a reductive complex Lie group is by definition one which can be realized as a complexification of maximal compact subgroup, this follows immediately from the above discussion.

It should be remarked that the quotient of a reductive complex Lie group by any reductive complex subgroup $H$ can be realized as a closed $G$-orbit in an appropriately chosen representation space. In particular, $G / H$ has the structure of an affine variety. Consequently, in the case at hand one refers to $X=G / K$ as an complex affine symmetric space.

Observe that $\tau:=\theta \sigma=\sigma \theta$ is an antilinear Lie algebra involution. Its fixed point algebra is denoted by $\mathfrak{g}_{0}$ with associated (noncompact) group $G_{0}$. Since $\theta$ commutes with $\tau$, it stabilizes $\mathfrak{g}_{0}$ and yields a decomposition

$$
\mathfrak{g}_{0}=\mathfrak{g}_{0}^{+}+\mathfrak{g}_{0}^{-}=\mathfrak{g}_{u}^{+}+i \mathfrak{g}_{u}^{-} .
$$

This decomposition is written as $\mathfrak{g}_{0}=\mathfrak{k}_{0}+\mathfrak{p}_{0}$ and the defining involution, which is the restriction of $\theta$ to $\mathfrak{g}_{0}$, is called the Cartan involution. Note that since $\mathfrak{p}_{0}$ consists of Hermitian matrices, $K_{0}$ is a maximal compact subgroup of $G_{0}$ and $G_{0} / K_{0}$ is a symmetric space of noncompact type.

Complex symmetric spaces are spherical. The fact that the symmetric space $X=G / K$ is spherical follows immediately from the Iwasawa decomposition of $\mathfrak{g}_{0}$. Starting from the above Cartan decomposition $\mathfrak{g}_{0}=\mathfrak{k}_{0}+\mathfrak{p}_{0}$ we now construct this decomposition.

First, let $\mathfrak{a}_{0}$ be a maximal Abelian subspace of $\mathfrak{p}_{0}$ and consider its action on $\mathfrak{g}_{0}$ by the adjoint representation. Since $\mathfrak{a}_{0}$ is contained in $\mathfrak{p}_{0}$ which consists of Hermitian operators in the given realization, the elements of $\mathfrak{a}_{0}$ are simultaneously diagonalizable over the reals. Note that the 0 -eigenspace of this action, i.e., the centralizer $\mathfrak{z}_{\mathfrak{g}_{0}}\left(\mathfrak{a}_{0}\right)$, splits as $\mathfrak{z}_{\mathfrak{g}_{0}}\left(\mathfrak{a}_{0}\right)=\mathfrak{m}_{0}+a_{0}$ where $\mathfrak{m}_{0} \subset \mathfrak{k}_{0}$.

An element $\alpha \in \mathfrak{a}_{0}^{*} \backslash\{0\}$ is called a root if

$$
\mathfrak{g}_{\alpha}:=\left\{\xi \in \mathfrak{g}_{0}: x(\xi)=\alpha(x) . \xi \text { for all } x \in \mathfrak{a}_{0}\right\}
$$


is nonempty. Let $\Phi$ denote the set of all roots. For $\alpha \in \Phi$ let $H_{\alpha}$ be the hyperplane $\{\alpha=0\}$ and define $P$ to be a component of the complement of $\cup_{\alpha} H_{\alpha}$. Fix some $x \in P$ and say that a root $\alpha \in \Phi$ is positive if $\alpha(x)>0$. Let $\Phi^{+}$denote the set of positive roots and

$$
\mathfrak{n}_{0}^{+}:=\oplus_{\Phi+} \mathfrak{g}_{\alpha} .
$$

Finally, observe that the dual action of $\theta$ maps positive roots to negative roots so that $\Phi=\Phi^{-} \dot{\cup} \Phi^{+}$and accordingly

$$
\mathfrak{g}_{0}=\mathfrak{n}_{0}^{-}+\mathfrak{m}_{0}+\mathfrak{a}_{0}+\mathfrak{n}_{0}^{+} .
$$

One checks that $\mathfrak{n}_{0}^{+}$and $\mathfrak{n}_{0}^{-}$are nilpotent Lie algebras. Since they are stabilized by $\mathfrak{a}_{0}$, it is immediate that $\mathfrak{a}_{0}+\mathfrak{n}_{0}^{+}$and $\mathfrak{a}_{0}+\mathfrak{n}_{0}^{-}$are solvable. For simplicity of notation we let $\mathfrak{n}_{0}:=\mathfrak{n}_{0}^{+}$.

\section{Theorem 4. (Iwasawa decomposition)}

$$
\mathfrak{g}_{0}=\mathfrak{k}_{0}+\mathfrak{a}_{0}+\mathfrak{n}_{0} .
$$

Proof. Note that $\theta\left(\mathfrak{n}_{0}^{+}\right)=\mathfrak{n}_{0}^{-}$so that we have a decomposition

$$
\mathfrak{n}_{0}^{-}+\mathfrak{n}_{0}^{+}=\delta^{-}+\delta^{+}
$$

where $\delta^{ \pm}$is the \pm 1 -eigenspace of $\theta$. Clearly $\mathfrak{p}_{0}=\mathfrak{a}_{0}+\delta^{-}$and $\mathfrak{k}_{0}=\delta^{+}+\mathfrak{m}_{0}$. Since $\delta^{+}\left(\operatorname{resp} . \delta^{-}\right)$is the diagonal (resp. antidiagonal) in $\mathfrak{n}_{0}^{+}+\mathfrak{n}_{0}^{-}$, the desired result follows.

By complexifying the above Iwasawa decomposition we have the decomposition

$$
\mathfrak{g}=\mathfrak{k}+\mathfrak{a}+\mathfrak{n}
$$

where $\mathfrak{k}=\operatorname{Fix}(\theta)$ and $\mathfrak{a}+\mathfrak{n}$ is a solvable Lie subalgebra of $\mathfrak{g}$.

Corollary 6. The homogenous space $X=G / K$ is spherical.

Proof. If $B$ is a Borel subgroup with Lie algebra containing $\mathfrak{a}+\mathfrak{n}$, then the $B$-orbit of the neutral point in $G / K$ is open.

\section{References}

1. A. Sawicki, A. Huckleberry, and M. Kuś. Symplectic geometry of entanglement. Comm. Math. Phys. 305, 441-468 (2011)

2. A. Sawicki and M. Kuś. Geometry of the local equivalence of states. J. Phys. A 44, 495301 (2011)

3. B. Kraus. Local Unitary Equivalence of Multipartite Pure States. Phys. Rev. Lett. 104, 020504, (2010)

4. V. Guillemin and S. Sternberg Symplectic techniques in physics. Cambridge University Press, Cambridge 1984.

5. M. Brion. Sur l'image de l'application moment. Lect. Notes Math. 1296, 177-192 (1987)

6. W. Dür, G. Vidal, and J. I. Cirac. Three qubits can be entangled in two inequivalent ways. Phys. Rev. A 62, 062314 (2000)

7. A. T. Huckleberry and T. Wurzbacher. Multiplicity-free complex manifolds. Mathematische Annalen 286, 261-280 (1990)

8. F. C. Kirwan. Cohomology of quotients in symplectic and algebraic geometry. Mathematical Notes vol 31. Princeton Univ Press, Princeton 1984

9. R. A. Horn C. R. Johnson. Matrix analysis. Cambridge University Press, Cambridge 1985

10. D. Akhiezer and E. B. Vinberg. Weakly symmetric spaces and spherical varieties. Trans. Groups 4, 3-24 (1999) 